\title{
Review of IoT-Based Wireless Sensors for Environmental Monitoring
}

\author{
Indu Mandwi ${ }^{1,}$ Sofia Pillai ${ }^{2}$, Mangala Madankar ${ }^{3}$, Piyush Ingole ${ }^{4}$ \\ ${ }^{1,2,3,4}$ G. H. Raisoni College of Engineering Nagpur, Maharashtra, India
}

\begin{abstract}
The recent adjustments in climate have multiplied the significance of environmental tracking, making it a topical and surprisingly active studies region. This Wi-Fi ld is based on remote sensing and on wireless sensor networks for gathering statistics about the environment. Current improvements, such as the vision of the internet of things (IoT), the cloud computing model, and cyberphysical structures, offer aid for the transmission and control of big amounts of information concerning the trends determined in environmental parameters. In this context, the present day paintings offers three specific IoT-primarily based Wi-Fi sensors for environmental and ambient tracking: one using user datagram protocol (UDP)-based totally wireless verbal exchange, one communicating thru Wi-Fi and hypertext transfer protocol (HTTP), and a 1/3 one using Bluetooth clever. All the offered systems provide the possibility of recording facts at far flung places and of visualizing them from each device with an internet connection, allowing the tracking of geographically massive regions. The improvement details of those systems are described, along with the predominant variations and similarities among them. the feasibility of the 3 developed structures for implementing monitoring packages, considering their power autonomy, ease of use, answer complexity, and net connectivity facility, turned into analyzed, and revealed that they make excellent applicants for IoT-primarily based solutions.
\end{abstract}

Keywords: Bluetooth, energy harvesting, IEEE 802.11 standards, Internet of things (IoT), low-power electronics

\section{Introduction}

The contemporary advances in the fields of generation and economic system are having a big effect over the environment, and have led to critical issues concerning pollution and weather trade. In the document released in 2014, the intergovernmental panel on climate alternate confirms that human sports are having an unequivocal and continuously growing have an effect on at the weather machine, with latest modifications that are exceptional over a long time to millennia [1]. On this context, environmental tracking represents a fundamental tool for accumulating applicable records about the atmosphere, new information and knowledge, and for in the end enforcing model and mitigation movements that deal with the degradation of the biosphere [2]. Every other related area currently gaining interest is the monitoring of the ambient in indoor areas. It has been shown that this is essential now not best for the consolation and health of constructing occupants [3], but also for the reduction of greenhouse gasoline emissions [4].The most common parameters being monitored consist of the temperature, humidity, rainfall, atmospheric stress, light intensity, air high-quality, stricken by pollutants which includes co2, co, sox, unstable organic compounds, and many others. One of the immediate blessings added via the purchase of such bodily proprieties, like soil moisture, temperature, and salinity, can be visible in agriculture, wherein sizeable water aid financial savings can be performed [5]-[7].

The majority of monitoring programs rely on WSNS, encouraged by using the indeniable blessings they bring: decrease costs because of the substitute of cables, variable community topologies, scalability, and decrease protection and commissioning expenses [8]. wireless sensors and sensor networks were effectively used in the implementation of solutions belonging to numerous fields, along with environmental monitoring [9]-[11], natural disaster prevention [12], modern consumption monitoring in big homes [13], monitoring systems for the symmetry of radiology operators in healthcare programs [14], [15], area tracking of humans, belongings or risky gases [16], [17], tools circumstance surveillance [18] and procedure manage [19] in business environments, and lots of others. The maximum widely used protocols in packages having less tight latency and reliability necessities, along with environmental monitoring, consist of zigbee (IEEE 802.15.4) [6], Wi-Fi (based on IEEE 802.11), and Bluetooth (IEEE 802.15.1). being primarily based on IEEE 802.15.4, a fashionable that gives low fees and low electricity at low information fees, zigbee is significantly used in a wide variety of tracking and manage software that require wireless connectivity. Those solutions provide electricity-green designs, but cannot observe tight latency and reliability requirements and require additional hardware for packaging facts and for transmitting them to the net. Wi-Fi is a popular networking era primarily based on the IEEE 802.11 set of requirements that offers higher transmission range and throughput compared to IEEE 802.15 .4 , with the value of higher power consumption. The development of low strength wireless devices, just like the rn131c/g from roving networks and the ESP8266 from espressif systems, made this era increasingly appealing for implementing Wi-Fi sensing packages. the sensors primarily based on this kind of modules take advantage of the prevailing infrastructure, have local IP-community compatibility, and might use protocols including user datagram protocol (UDP) and hypertext switch protocol (HTTP), disposing of the want for applications that encapsulate the information for transmitting them to the net. Bluetooth low strength (BLE), or Bluetooth smart, turned into delivered in 2010 with the intention of extending the application area of Bluetooth to strength-constrained devices, such as Wi-Fi sensors, and has visible a non-stop evolution for the reason that then. But, the implementation of tracking 


\section{International Journal of Science and Research (IJSR) \\ ISSN (Online): 2319-7064}

Index Copernicus Value (2016): 79.57 | Impact Factor (2015): 6.391

programs with internet connectivity requires the usage of Bluetooth net gateways for sending and receiving dimension statistics. In industrial settings, where heavy interference are present, and the reliability of information transmission, high replace fees, determinism, and high availability are of super importance, the wirelesshart (IEC62591), ISA100 wireless (IEC62734), and the IEEE 802.15.4.e layer 1 and a couple of preferred are used. Those provide reliable and at ease lowstrength Wi-Fi operation for noncritical tracking and manipulate packages. Sensor networks rely on the net for transmitting facts to valuable stations, wherein similarly processing may be performed on the received information. The placement of sensors in locations situated at big distances from the server or the bottom station, taking benefit of the net, permits the collection of environmental information from big geographical regions. This is made viable through development of latest transceivers with longer degrees and lower electricity intake, which could establish internet connections.

This paper presents the layout information, the development, and the analysis of 3 unique sensors that enable the success of net connected answers for monitoring the environment or the ambient at far flung locations: one employing UDPprimarily based wireless communication, one based totally at the HTTP protocol, and one consisting in electricityharvesting BLE beacons. being supplied with internet connection capabilities, the advanced sensors represent a part of the internet of things (IoT), the imaginative and prescient that "permits human beings and matters to be related each time, anyplace, with whatever and each person, ideally the usage of any direction/network and any service". visible as the forth wave of computing, the IoT is starting to broaden at an expanded pace and it's far believed that within the yr 2021, more than 25 billion gadgets can be linked to the internet. The analysis of the 3 answers supplied here highlights the truth that they constitute possible options for zigbee-primarily based devices intensively utilized in monitoring packages.

\section{Related Work}

The literature has pronounced a big variety of studies efforts that employ Wi-Fi sensors for reaching tracking applications. donno et al. propose a solution where self-powered radio frequency identity tags, ready with temperature, light, and acceleration sensors, are used. The tool has the capacity to reap RF power and its operation has been validated via two actual-world experiments, in which the obtained facts are gathered with the aid of a host PC with the help of a reader antenna. The layout achieved a transmission range of up to 10 and $20 \mathrm{~m}$ in completely passive and battery-assistedpassive modes, respectively. The proposed device may be used for tracking the ambient or outside climate parameters, and, if the host computer is supplied with an internet connection, can be a part of an IoT-primarily based solution. Reference offers an open source Wi-Fi mesh internetworking module for environmental monitoring, with the purpose of promoting this type of routing in such packages. This is based on a low-fee RF transceiver, with greater compact and much less complicated code than the only of a zigbee layout, and works in connection with a sensor node. the system was compared to an off-the-shelf product, xbee, with the realization that it could provide similar or maybe better performance than business gadgets. other systems for tracking programs belonging to distinctive fields, all based totally on zigbee, are pronounced in [6], [13], and. The main drawback of those is composed within the requirement for a gateway.

Numerous monitoring answers primarily based on BLE technology have regarded and are gaining ground mainly in home automation, after its creation in 2010. Through imparting low power, low fee, and decreased device dimensions, the authors agree with that this era has a high potential of becoming essential for both the IoT and for smart houses. this fashion will also be sustained by means of the availability of native guide supplied by way of present day cell devices, in comparison to IEEE 802.15.4, so as to additionally reduce the cost of BLE gadgets. The simulation consequences display that this method contributes to the reduction of peak load demand and electricity intake prices, in the long run main to economic financial savings. Furthermore, it has been shown that the overall performance of the proposed BLE community is better than the one received in the case of IEEE 802.15.4 in terms of packet shipping ratio, delay, and jitter. With the continuous enhancements introduced to the protocol, which include the help for mesh networking, and the extension of the variety presented, it is believed that this technology could be taken into consideration for implementing environmental monitoring programs.

\section{Solution based on Wi-Fi}

The two sensors that communicate by use of Wi-Fi generation are based totally at the identical hardware, the difference among the two consisting in the protocol that become used, particularly UDP or HTTP. The general architecture of the devices based on wireless generation is supplied in fig. 1. it consists of the application processor, a CY8C3246PVI-147 programmable device on chip microcontroller (PSOC3) produced with the aid of cypress semiconductor, a wireless nearby area community (lan) module, $\mathrm{RN}-131 \mathrm{c} / \mathrm{g}$, and the temperature and relative humidity sensor (SHT21), all powered with the aid of a three VCR123a 1500MAH battery. The selection of the usage of a separate application processor removes the possibility of interfering with the verbal exchange stack on the WLAN module. Consequently, the processor in the developed devices is in price with appearing all the moves for the proper operation of the tool, namely, power management, acquisition of statistics from the sensing unit, and communication. For transmitting the records to a base station, a serial hyperlink among the PSOC three device and the communication module, and an API (utility programming interface), known as wifly, are used. The incentive for deciding on the $\mathrm{RN}-131 \mathrm{c} / \mathrm{g}$ Wi-Fi module is composed in its low-electricity operation, providing $4 \mu \mathrm{a}$ at some stage in sleep and quick 210MA pulses at some stage in transmission. The improvement of different inexpensive wireless modules based on the IEEE 802.11 set of standards, inclusive of the 


\section{International Journal of Science and Research (IJSR) \\ ISSN (Online): 2319-7064}

Index Copernicus Value (2016): 79.57 | Impact Factor (2015): 6.391

ESO8266 from express if, will multiply the variety of feasible answers. however, with a contemporary large than $20 \mu \mathrm{a}$ in deep sleep mode, more modern tactics for accomplishing energy efficiency inside the designs using it's going to ought to be investigated. The prototype of the developed Wi-Fi sensor is supplied in fig. 2

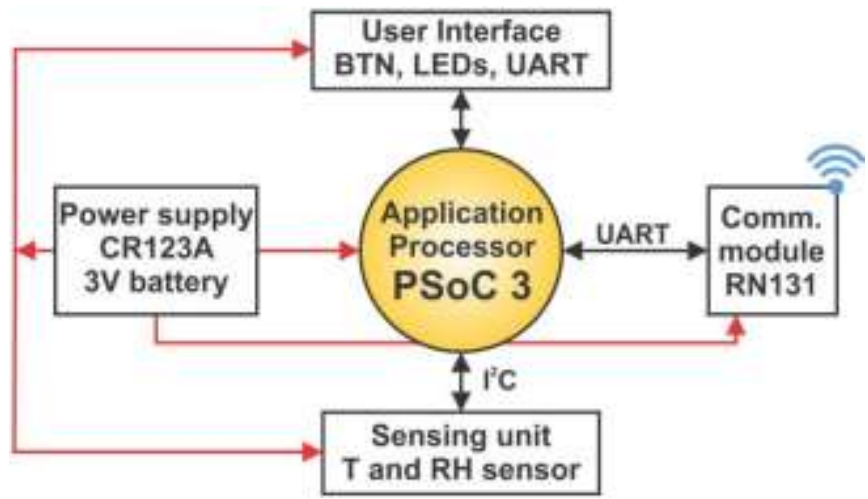

Figure 1: Wi-Fi environmental sensor block diagram

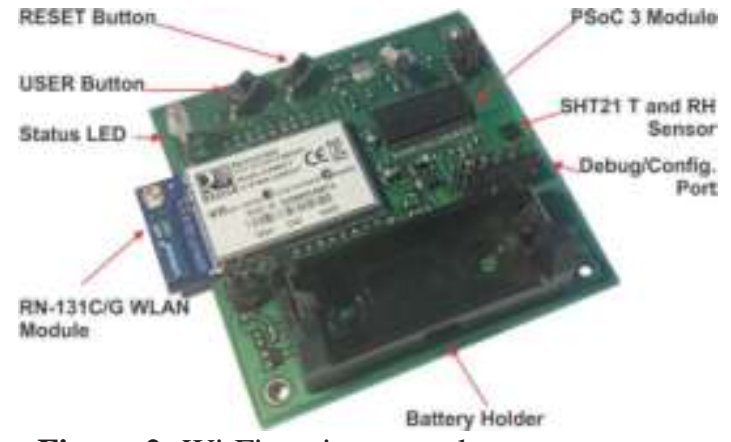

Figure 2: Wi-Fi environmental sensor prototype.

\section{A. UDP}

Fig. 3 affords an UDP-based totally cyber-bodily machine for tracking the temperature and relative humidity in the surroundings or ambient. This takes advantage of the existing IEEE 802.11 infrastructure for sending measurement statistics to a cloud-based server platform and presents the opportunity of visualizing the facts from each tool with an internet connection. The usage of UDP allows the lowstrength operation of the Wi-Fi sensors, due to its connectionless nature. Furthermore, this protocol gives decrease packet sizes, increased speeds and coffee latency, in comparison to tcp/ip. All these come at a charge, namely, a loss in transmission reliability, due to the fact there may be no renowned message received for the packets being sent. the server platform can live within the cloud, or may be an UDP listener walking on a computer, that could interpret the acquired information, keep them in a database, and provide the possibility of visualizing and processing them in line with the user's needs, via a web server.

Due to the fact there are numerous sensors attached to the tool, the structure is more complex than the overall one, presented in fig. 1. this one consists of a separate strength supply for the sensors, that may be grew to become off, and ends in a energy intake of most effective $10 \mu \mathrm{a}$ throughout sleep durations. Through placing the ratio between wakeup durations and sleep periods, the device can reach a power intake among a hundred and $200 \mathrm{mw}$. The intake profile of the tool, in this case, is particularly prompted by way of the sensors connected, particularly by using the carbon dioxide sensor, which calls for the use of a virtual filter for attenuating the noise that adds time to the wakeup period. For counteracting these shortcomings, other optimizations have to be accomplished. In this direction, the hobby of the RF transceiver needs to be kept to a minimum through the sending of best brief messages, which include numerous pairs of hexadecimal numbers, opcodes, to the server platform. This lead to a packet size of $76 \mathrm{~b}$. The power signature of the tool for the duration of a wakeup period, with the filter of the $\mathrm{CO} 2$ sensor set to 2, leading to an energetic time length of seven $\mathrm{s}$, a wakeup period comparable with the one of the HTTP-primarily based

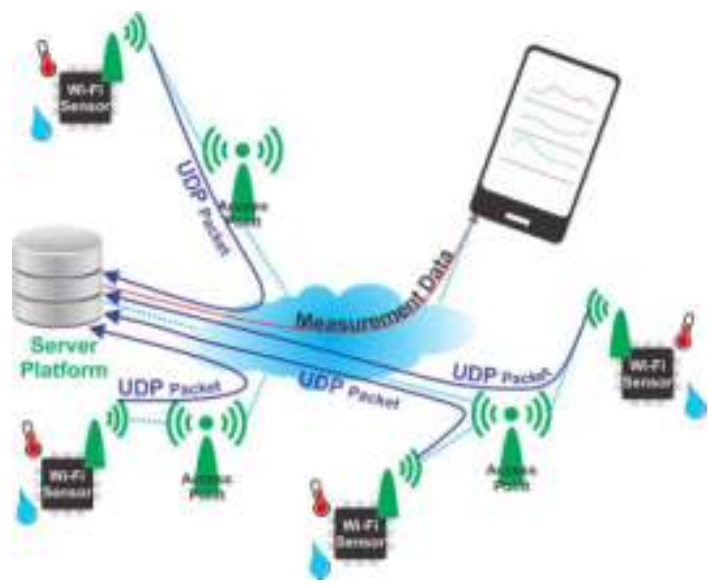

Figure 3: Environmental monitoring system with communication based on UDP.

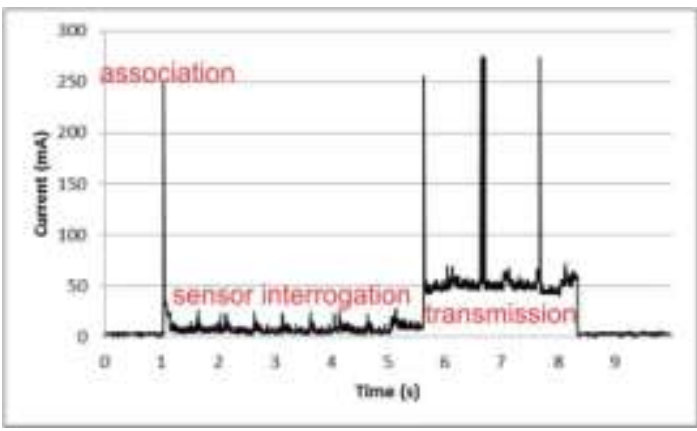

Figure 4: Complete wakeup period of UDP-based device$\mathrm{T}, \mathrm{RH}, \mathrm{CO} 2$, pressure, and light sensors attached.

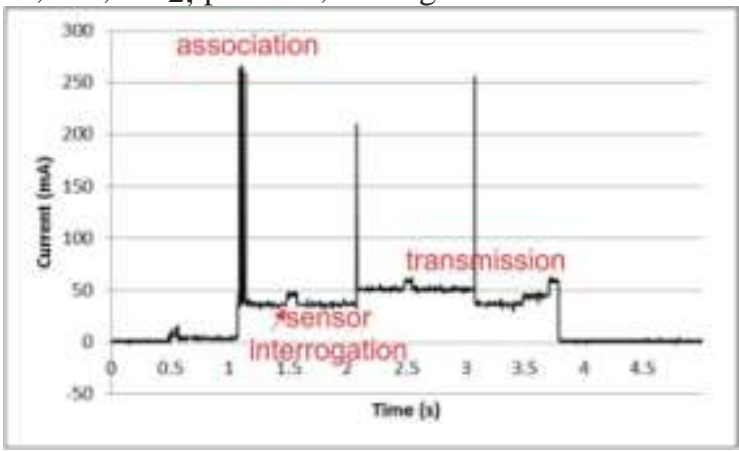

Figure 5: Complete wakeup period of UDP-based device-SHT21 sensor attached.

It is offered in Fig. 4. The average current on modern-day right here is $25.03 \mathrm{~mA}$.

\section{Volume 6 Issue 12, December 2017 www.ijsr.net}




\section{International Journal of Science and Research (IJSR) \\ ISSN (Online): 2319-7064}

Index Copernicus Value (2016): 79.57 | Impact Factor (2015): 6.391

Based on the consumption all through wakeup and energetic intervals, and on the leakage currents of the components that make up the machine, an anticipated life of approximately 36 months, period similar to the one supplied through ZigBee based totally solutions, is reached for a size cycle of $1 \mathrm{Hr}$.

Fig. 5 affords the electricity profile of a wakeup cycle of the device presented in Figs. 1 and 2. This has only an SHT21 sensor attached, a wakeup period of $3.5 \mathrm{~s}$, and a decrease packet length, of 52Byte, with a mean modern drawn of 37.0 $\mathrm{mA}$. With an average sleep cutting-edge less than $20 \mu \mathrm{A}$, this could lead to a battery lifetime exceeding three years, on a 60 min size cycle.

The experiments finished for comparing the package loss ratio of UDP-based totally sensors highlighted that the devices may be used in applications for environmental tracking, wherein excessive reliability isn't always crucial. For a tool being set to degree temperature and relative humidity values every minute, $3.29 \%$ unmarried packets, respectively $0.18 \%$ consecutive packets have been misplaced, from a total of 3000 messages being dispatched $(95.4 \%$ a hit transmission charge). For a size cycle of $10 \mathrm{~min}$ and four hundred messages dispatched, $4.34 \%$ unmarried packet, and $0.26 \%$ consecutive packet losses happened $(95.4 \%$ a hit transmission price). The experiments finished suggest that those losses are because of the community itself and with the aid of the protocol getting used, and are not associated with the layout of the wireless devices or to the cloud platform.

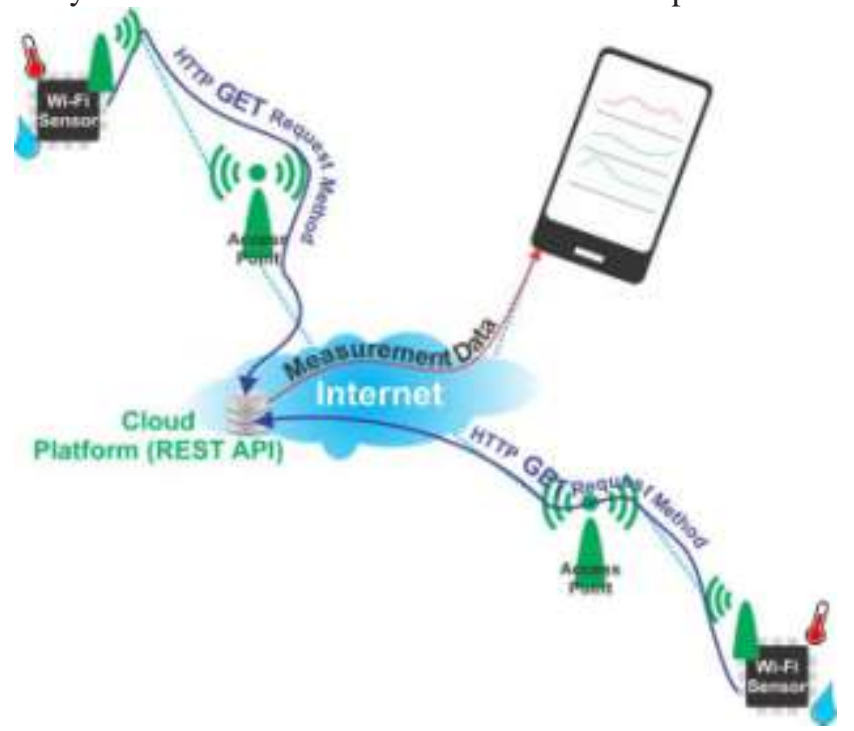

Figure 6: Environmental monitoring system with communication based on HTTP requests

\section{B.HTTP}

Sensors that connect with a WLAN and that send size statistics through HTTP requests have been designed and manufactured. These operate in a state of affairs just like the one supplied in Fig. 6. It was predicted that the use of this utility protocol might increase the reliability of statistics transmission, with the aspect impact of additionally growing the energy intake of the gadgets. The operation of the Wi-Fi sensor is just like the only presented in the previous segment, the most effective distinction consisting within the way in which facts are transmitted. Here, in the course of the wakeup duration, the tool interrogates the attached sensor, opens an
HTTP connection, and sends the statistics in the shape of a GET request. After receiving the reaction from the server, it closes the connection and is going to sleep, in line with the length set via person. For checking out the tool operation, a time-honored free public cloud provider became selected, facts.sparkfun.com, which affords the opportunity of logging facts from remote places, in line with the IoT imaginative and prescient. The facts are posted right here via using GET and post requests, based on a public key and on a non-public key provided by the cloud platform whilst creating a facts movement. These can be accessed from everywhere and the associated records can be retrieved in exclusive codecs (JavaScript item Notation, comma separated values report, established question Language) and similarly processed, if essential. In the equal manner as for the previous tool, this one can be configured with the aid of the user via a serial interface and a menu, allowing the putting of the size duration, of WLAN connectivity parameters, and of the far away host related facts, inclusive of the deal with, and public and private key requested for posting statistics. Due to the fact the application isn't always coping with touchy facts, and because the best and most efficient solutions was preferred, GET requests have been used, which permit the passing of information through the Uniform resource Locator. The experiments indicated a duration of about $6 \mathrm{~s}$ for a successful facts transmission to the far off host, as Fig. 7 indicates, at some stage in which the common contemporary drawn by means of the device is of $41.20 \mathrm{~mA}$. Knowing this, the capability of the battery used, and the average sleep current drawn (less than $20 \mu \mathrm{A}$ ), an estimation of the battery lifetime using a $1 \mathrm{~min}$ cycle changed. This indicated a theoretical working time of $297 \mathrm{~h}$, and the completion of 17.822 dimension and transmission cycles. For measuring the real energy consumption of the device in a state of affairs as near fact as possible, and for validating the estimation, it turned into programmed to ship also the voltage of the battery except temperature and relative humidity values. After this, the device was set to send facts every minute and left to ship information till it stops running due to the depletion of the battery. The discharge curve that becomes received may be shown in Fig. 8.

This suggests a number of 15183 successful transmissions from a total of 15725, and an operating length of $262 \mathrm{~h}$ on a $3 \mathrm{~V}, \mathrm{CR} 123 \mathrm{~A}$ battery. These facts lead to an expected lifetime of approximately eleven months using a dimension cycle of an hour. The success transmission ratio obtained in this situation is of $96.56 \%$, cost affected additionally with the aid of negative net connectivity of the get right of entry to point for multiple hours. Fig. 9 gives the temperature and relative humidity graph acquired within the testing phase, in a actual operation situation, over a length of 11 days.

\section{Volume 6 Issue 12, December 2017




\section{International Journal of Science and Research (IJSR) \\ ISSN (Online): 2319-7064}

Index Copernicus Value (2016): 79.57 | Impact Factor (2015): 6.391

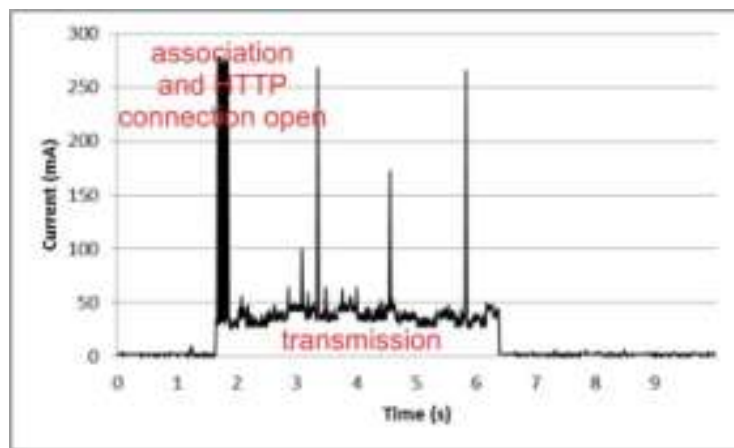

Figure 7: Complete wakeup period of HTTP-based device-SHT21 sensor attached

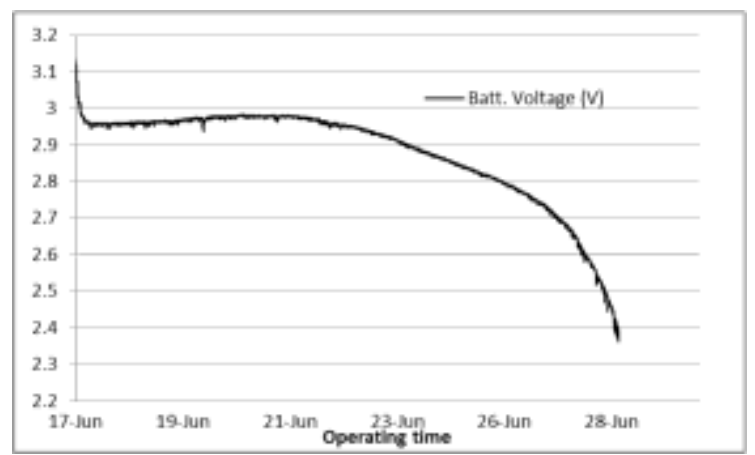

Figure 8: Battery discharge curve during testing

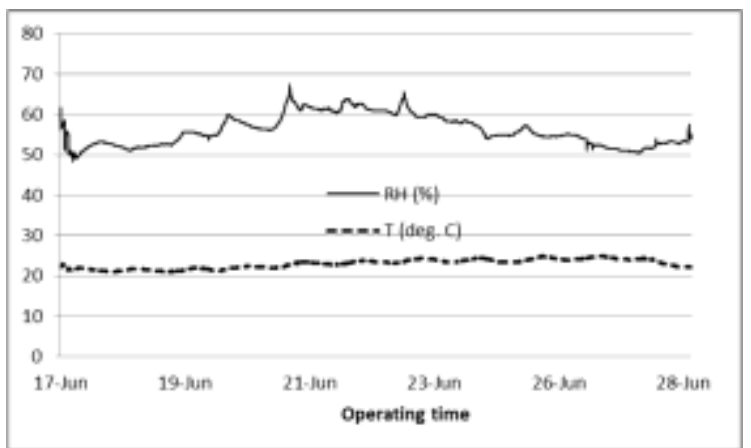

Figure 9: Temperature and relative humidity chart obtained during testing.

\section{Solution based on BLE}

Bluetooth smart is getting an increasing amount of interest recently, and, consequently, a solution employing this generation became also advanced. However, the state of affairs in this situation is a very distinctive one, as provided in Fig. 10. The gateway in this case is a mobile one, and may be a phone that receives the facts while its miles located inside the sensor's variety. From here, the information may be sent to a server. The use of the cell device's connectivity skills. Via the use of the area obtained by the cellular gateway, geographical facts and the time may be introduced to the recorded values, increasing their complexity and relevance. One simple instance consists within the success of a smart town's map showing the changes inside the environmental parameters, including temperature or air, over the years. This will mean the deployment of huge numbers of BLE sensors in exceptional places and history software strolling at the citizen's smart phones, appearing as cellular forwarders of information to the cloud.
In this route, a BLE beacon that periodically measures the temperature and relative humidity within the air, the atmospheric pressure, and the light depth became developed (Fig. 11). The wireless sensor polls the sensors every one minute and pronounces BLE commercials containing the acquired records every $3 \mathrm{~s}$. The application running at the mobile gateway constantly scans for BLE commercial packets and, if in range of one of the beacons, it gets and strategies them. The application can show the values measured by means of the connected sensors regionally or are able to ship them to a cloud platform in an IoT state of affairs, as offered in Fig. 10.

The developed BLE beacon is supplied in Fig 11. Its principal additives installed at the published circuit board include a CYBLE-022001-00 programmable radio on chip module assisting BLE wireless communication (EZ-BLE PROC Module), produced through Cypress Semiconductor, an SHT21 temperature and relative humidity sensor, an MPL115A2, absolute pressure sensor, an OPT3001 virtual ambient light sensor, a BQ25504 ultra low-power improve converter with battery control for strength harvester applications, and sun cells for extending the operation of the tool and the battery lifetime. Two separate electricity components are used, one for the CPU and Bluetooth chip and the other for the sensors. For decreasing the energy intake, the latter can be grown to become off, while the sensors aren't in use. The application running on the BLE Module instantiates a BLE familiar get admission to profit broadcaster that advertises the temperature, relative humidity, atmospheric strain, and mild intensity values in non connectable advertising mode. A majority of these values are read thru the $\mathrm{I} 2 \mathrm{C}$ bus to which all of the sensors are connected. The environmental values are constantly updated, as quickly as a brand new size is carried out. The tool wakes up each $3 \mathrm{~s}$, for sending a commercial packet, and takes the measurements from the sensors the usage of the communication interface in each minute. The application running on the mobile gateway continuously scans for BLE advertisement packets and, if in range of one of the beacons, it receives and processes them. The application can display the values measured by the attached sensors locally or is able to send them to a cloud platform in an IoT scenario, as presented in Fig. 10.

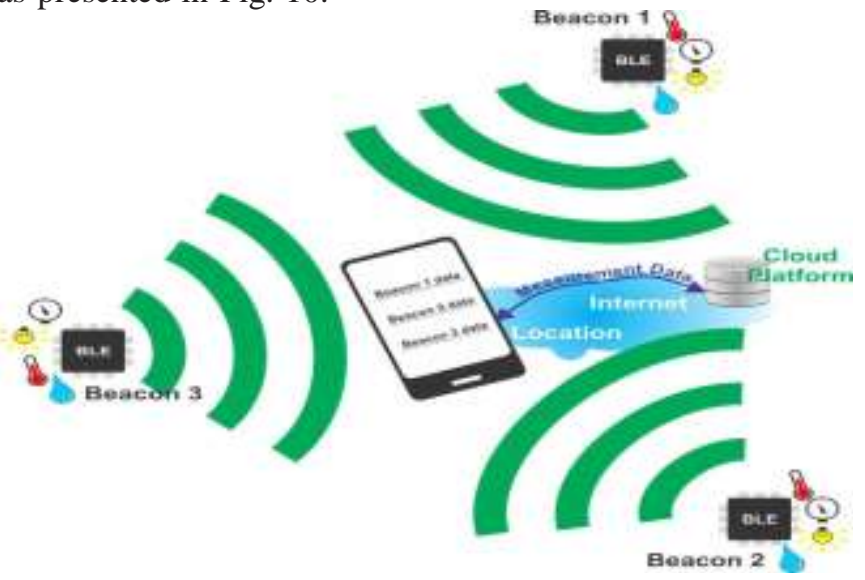

Figure 10: Environmental monitoring system based on BLE.

Volume 6 Issue 12, December 2017 www.ijsr.net 


\section{International Journal of Science and Research (IJSR) \\ ISSN (Online): 2319-7064}

Index Copernicus Value (2016): 79.57 | Impact Factor (2015): 6.391

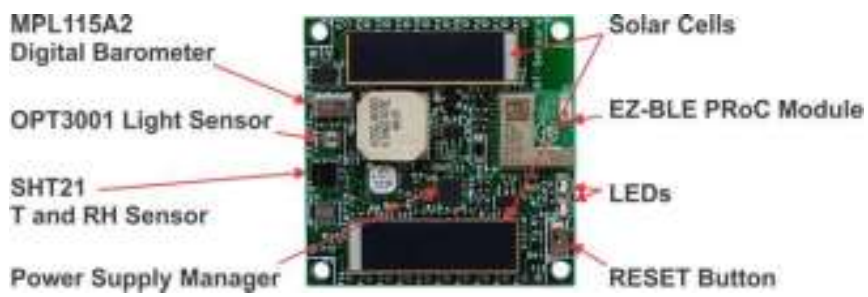

Figure 11: BLE environmental beacon prototype.

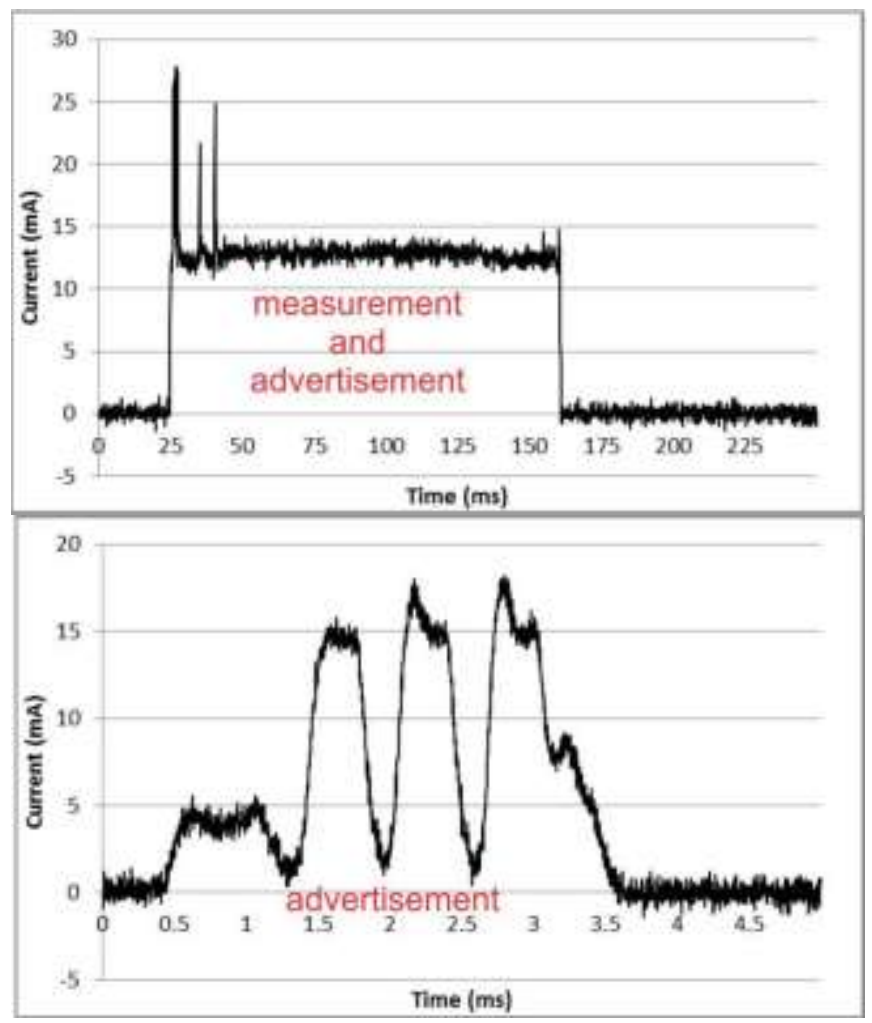

Figure 12: BLE device power consumption in measurement (top graph) and advertising (bottom graph) mode.

The application running on the mobile gateway continuously scans for BLE advertisement packets and, if in range of one of the beacons, it receives and processes them. The application can display the values measured by the attached sensors locally or is able to send them to a cloud platform in an IoT scenario, as presented in Fig. 10.

The application running on the BLE Module instantiates a BLE generic access profit broadcaster that advertises the temperature, relative humidity, atmospheric pressure, and light

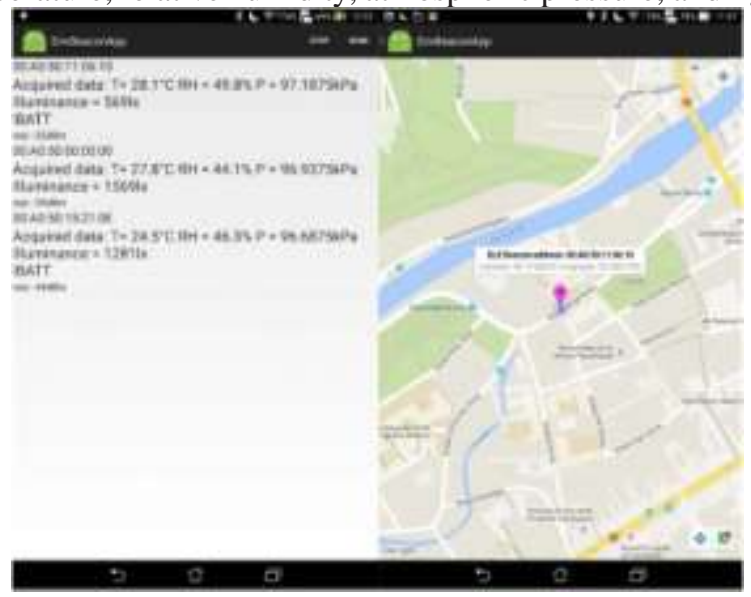

Figure 13: BLE smart phone application
Values in non connectable advertising mode. All these values are read through the $\mathrm{I}^{2} \mathrm{C}$ bus to which all the sensors are connected. The environmental values are continuously updated, as soon as a new measurement is performed. The device wakes up every $3 \mathrm{~s}$, for sending an advertisement packet, and takes the measurements from the sensors using the communication interface every minute. After the device acquires the data from the attached sensors, it updates the advertisement payload with new data, advertises, and goes back to low power mode, for minimizing the power consumption. The data are stored in the Manufacturer Specified Data field $(0 \mathrm{xFF})$ of the advertisements, as integer values on two bytes. An additional byte is added for signaling the status of the accumulator, indicating the event in which the voltage goes under a previously set lower limit.

The initial analysis of the power consumption of the developed BLE beacon indicates that it is able to assure the power levels required for stand-alone operation, with the solar cells being capable of charging the accumulator within a single sunny day. Fig. 12 presents the current drawn by the device during the initial phase of testing and evaluation of the developed device. The average current is $12.7 \mathrm{~mA}$ for a period of $140 \mathrm{~ms}$, when communicating with the sensors and sending an advertisement packet. A single advertisement packet is sent in a $3 \mathrm{~ms}$ time frame, with an average current of $7.8 \mathrm{~mA}$. The first manufactured prototype consumes below $40 \mu \mathrm{A}$ in sleep mode.

The Android application, that can run on mobile devices, implements a GATT (Generic Attribute Profile) observer with the following functions: the scanning of BLE advertisements from devices in the vicinity for receiving the data; the display of the received data under a list form and their storage into a cloud platform; the visualization of location coordinates (latitude, longitude), associated with the data, on a Google map. The environmental data acquired from the beacons, displayed in a list item, can be sent to a cloud platform using HTTP POST or GET requests. Fig. 13 presents the graphical user interface of the application running on mobile devices, acting as gateways.

\section{Conclusion}

Three diverse remote sensors for executing IoT-based answers for natural checking were outlined, created, and broke down. Every one of them are created utilizing business off-the-rack discrete parts and give simple access to the Internet utilizing at least extra equipment and programming assets. The examination of the three usage uncovered the way that Wi-Fi and BLE are two advances suited for checking applications that can effectively contend with the generally utilized ZigBee convention. Not surprisingly, Wi-Fi devours more vitality however empowers the advancement of arrangements with decreased aggregate cost of possession using the current framework. The third arrangement, utilizing Bluetooth Smart communication, has additionally been turned out to be productive, and the promising outcomes empower the improvement of frameworks in light of this

\section{Volume 6 Issue 12, December 2017 www.ijsr.net}




\section{International Journal of Science and Research (IJSR) \\ ISSN (Online): 2319-7064}

Index Copernicus Value (2016): 79.57 | Impact Factor (2015): 6.391

innovation. Moreover, the refinement of the convention and the expansion of work organizing abilities to BLE gadgets will make it considerably more appealing for executing such arrangements. The examination of the created frameworks additionally demonstrate the way that, with the accessibility of progressively vitality effective transmission modules, control reaping is pertinent likewise to $\mathrm{Wi}-\mathrm{Fi}$ innovation, as of not long ago thought to be excessively control hungry for planning remote sensors.

\section{References}

[1] R. K. Pachauri and L. A. Meyer, Eds., Climate Change 2014: Synthesis Report. Contribution of Working Groups I, II and III to the Fifth Assessment Report of the Intergovernmental Panel on Climate Change [Core Writing Team, IPCC, Geneva, Switzerland, 2014, p. 151.

[2] M. Harris, "Mules on a mountain," IEEE Spectr., vol. 53, no. 6, pp. 50-56, Jun. 2016.

[3] L. Zhang and F. Tian, "Performance study of multilayer perceptrons in a low-cost electronic nose," IEEE Trans. Instrum. Meas., vol. 63, no. 7, pp. 1670-1679, Jul. 2014.

[4] S. Mukhopadhyay, "Research activities on sensing, instrumentation, and measurement: New zealand perspective," IEEE Instrum. Meas. Mag., vol. 19, no. 2, pp. 32-38, Apr. 2016.

[5] J. Gutierrez, J. F. Villa-Medina, A. Nieto-Garibay, and M. A. Porta-Gandara, "Automated irrigation system using a wireless sensor network and GPRS module," IEEE Trans. Instrum. Meas., vol. 63, no. 1, pp. 166-176, Jan. 2014.

[6] N. Harris, A. Cranny, M. Rivers, K. Smettem, and E. G. Barrett-Lennard, "Application of distributed wireless chloride sensors to environmental monitoring: Initial results," IEEE Trans. Instrum. Meas., vol. 65, no. 4, pp. 736-743, Apr. 2016.

[7] H.-H. Lin et al., "An open-source wireless mesh networking module for environmental monitoring," in Proc. IEEE Int. Instrum. Meas. Technol. Conf. (IMTC), May 2015, pp. 1002-1007.

[8] M. T. Lazarescu, "Design of a WSN platform for longterm environmen- tal monitoring for IoT applications," IEEE J. Emerg. Sel. Topics Circuits Syst., vol. 3, no. 1, pp. 45-54, Mar. 2013.

[9] O. Postolache, J. D. Pereira, and P. S. Giral̀ fo, "Wireless sensor network- based solution for environmental monitoring: Water quality assessment case study," IET Sci., Meas. Technol., vol. 8, no. 6, pp. 610-616, 2014.

[10] Y. Liu, Y. He, M. Li, J. Wang, K. Liu, and X. Li, "Does wireless sensor network scale? A measurement study on GreenOrbs," IEEE Trans. Parallel Distrib. Syst., vol. 24, no. 10, pp. 1983-1993, Oct. 2013.

[11]G. Werner-Allen et al., "Deploying a wireless sensor network on an active volcano," IEEE Internet Comput., vol. 10, no. 2, pp. 18-25, Mar. 2006.

[12] J. P. Amaro, R. Cortesão, J. Landeck, and F. J. T. E. Ferreira, "Harvested power wireless sensor network solution for disaggregated current estimation in large buildings," IEEE Trans. Instrum. Meas., vol. 64, no. 7, pp. 1847-1857, Jul. 2015.

[13]D. Magalotti, P. Placidi, M. Paolucci, A. Scorzoni, and L. Servoli, "Experimental characterization of a wireless personal sensor node for the dosimetry during interventional radiology procedures," IEEE Trans. Instrum. Meas., vol. 65, no. 5, pp. 1070-1078, May 2016.

[14] D. Magalotti, P. Placidi, M. Dionigi, A. Scorzoni, and L. Servoli, "Experimental characterization of a personal wireless sensor network for the medical X-ray dosimetry," IEEE Trans. Instrum. Meas., vol. 65, no. 9, pp. 2002-2011, Sep. 2016.

[15]M. C. R. Talampas and K. S. Low, "An enhanced geometric filter algorithm with channel diversity for device-free localization," IEEE Trans. Instrum. Meas. vol. 65, no. 2, pp. 378-387, Feb. 2016.

[16] M. L. Cao, Q. H. Meng, Y. Q. Jing, J. Y. Wang, and M. Zeng, "Distributed sequential location estimation of a gas source via convex combination in wsns," IEEE Trans. Instrum. Meas., vol. 65, no. 6, pp. 1484-1494, Jun. 2016.

[17] M. D. Prieto, D. Z. Millan, W. Wang, A. M. Ortiz, J. A. O. Redondo, and

[18] L. R. Martinez, "Self-powered wireless sensor applied to gear diagnosis based on acoustic emission," IEEE Trans. Instrum. Meas., vol. 65, no. 1, pp. 15-24, Jan. 2016.

Volume 6 Issue 12, December 2017

www.ijsr.net

Licensed Under Creative Commons Attribution CC BY 